\title{
Gestaltungsbereiche der Digitalen Transformation von Unternehmen: Entwicklung eines Reifegradmodells
}
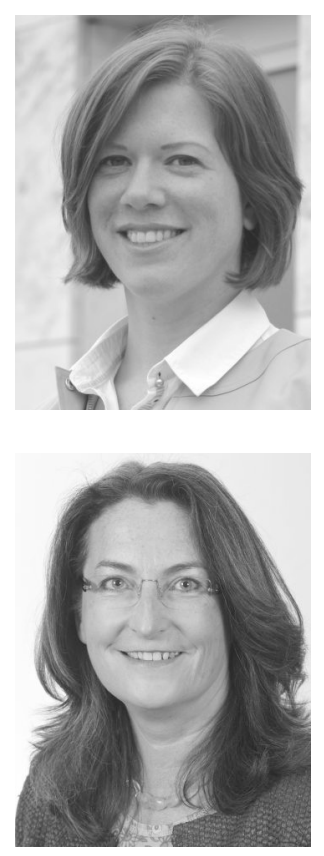

Sabine Berghaus, Andrea Back

Digitale Transformation; Organisationale Transformation; Reifegradmodell; Digitale Strategie; Transformationsmanagement

digital transformation; organizational transformation; maturity model; digital strategy; transformation management

Die dynamische Entwicklung von neuen digitalen Technologien stellt Unternehmen vor die Herausforderung, die Geschäftsmodelle, Produkte und Prozesse an die neue digitale Realität anzupassen. Die digitale Transformation von Unternehmen ist ein disruptiver Prozess, den Entscheidungsträger strategisch einleiten und aktiv gestalten. In dieser Studie wird zu diesem Zweck mit einem Literatur-Review, qualitativen Interviews und zwei Fokusgruppen ein Reifegradmodell entwickelt, das eine ganzheitliche Betrachtungsweise der Gestaltungsbereiche für die digitale Transformation ermöglicht. Das Modell besteht aus neun Dimensionen, die in einem Kriterienkatalog mit 59 Indikatoren präzisiert sind. Das Ergebnis dieser Studie ermöglicht Praktikern eine Analyse ihrer Aktivitäten im Zusammenhang mit der digitalen Transformation des Unternehmens und zeigt weitere Forschungsfelder auf.

The dynamic proliferation of digital technologies challenges organizations to adapt their business models, products and processes to the new digital reality. The digital transformation of organizations is a disruptive process that decision makers strategically develop and actively carry out. Based on a literature review, qualitative interviews and two focus groups, this study proposes a maturity model that allows a holistic view on the areas affected by digital transformation. The model consists of nine dimensions that consist of a criteria list with 59 indicators. The contribution of this study enables practitioners to assess their activities associated with the digital business transformation and presents further areas of research.

\section{Kontext der digitalen Transformation}

Die dynamische Entwicklung von neuen digitalen Technologien fordert Unternehmen heraus, ihre Geschäftsmodelle, Produkte und Prozesse an die neue digitale Realität anzupassen (Hansen et al. 2011; Berman 2012). Transformationsprozesse von Unternehmen, die durch neue Informationstechnologien (IT) ermöglicht wurden, sind kein neues Thema und wurden in der Vergangenheit breit untersucht. Forschungsobjekte waren dabei beispiels- 
weise der Zusammenhang von IT-Fähigkeiten und Unternehmenserfolg (Bharadwaj 2000; Chae et al. 2014), der Einfluss von Innovationen von Informationssystemen auf Organisationen (Swanson 1994), die Entwicklung von organisationalen Fähigkeiten durch IT (Brynjolfsson/Hitt 2000) oder die gemeinsame Ausrichtung von IT- und Unternehmensstrategie (Henderson/Venkatraman 1993; Chen et al. 2010; Drnevich/Croson 2013).

Unter digitaler Transformation werden Veränderungen in verschiedenen Bereichen wie Technologie, Produkten, Dienstleistungen, Customer Experience, Unternehmensprozessen oder Geschäftsmodellen durch digitale Technologien zusammengefasst (Markides 2006; Westerman et al. 2011). Die rapide Entwicklung von digitalen Technologien und daraus resultierende Innovationen haben Unternehmen und ganze Branchen disruptiv verändert (Govindarajan/Kopalle 2006; Cravens et al. 2009; Moreau 2013; Green/Cluley 2014). Gal et al. (2007) charakterisieren disruptive Innovationen durch drei Merkmale: Erstens heben sie sich deutlich von anderen Alternativen ab. Dies wird darin sichtbar, dass digitale Technologien neue Anwendungsfälle ermöglichen, wie beispielsweise der Einsatz von ortsbezogenen Diensten durch mobile Applikationen (Sammer et al. 2013). Zweitens erfordert ihre Anwendung den Erwerb von neuen Denkmustern. Diese werden erforderlich, da die Allgegenwärtigkeit von Technologie das Nutzerverhalten und die Nutzererwartungen an Produkte und Services ändert (Brenner et al. 2014). Drittens haben sie Einfluss auf zukünftige Innovationen, angrenzende Strukturen und Prozesse. Die allgegenwärtige Nutzung von Webtechnologie ermöglicht die Generierung und Nutzung von mehr Daten, welche wiederum analysiert und eingesetzt werden können, um sowohl das Kundenerlebnis als auch Geschäftsprozesse zu verbessern (Mithas et al. 2013a; Sharma et al. 2014). Der Einfluss von digitalen Innovationen auf Prozesse und Strukturen wird im Unternehmenskontext ebenfalls in der veränderten Rolle der CIOs (Weill/Woerner 2013) sichtbar, die stärker mit Fachbereichen zusammenarbeiten müssen.

\section{Reifegradmodelle zur Gestaltung von Transformationsprozessen}

Diese Vielzahl von Veränderungen erfordert, dass Unternehmen Strukturen, Prozesse, Produkte und Kultur ganzheitlich transformieren (Österle 2007) und die daraus resultierende Komplexität bewerkstelligen (Beese et al. 2015). Das schnelle Tempo der Digitalisierung führt dazu, dass diese Transformation von Unternehmen eher eine radikale Erneuerung als eine schrittweise Verbesserung ist (Lyytinen/Rose 2003; Bourreau et al. 2012) und andere Innovationsprozesse gewählt werden als bisher (Henfridsson/Yoo 2014). Die digitale Transformation ist ein Prozess, den Unternehmen strategisch einleiten und aktiv gestalten (Besson/Rowe 2012). Zu diesem Zwecke ist es notwendig, dass Entscheider die eigene Organisation sowie die Entwicklungen und das Marktumfeld genau kennen (Tornatzky et al. 1990) und basierend auf diesem Wissen ein strategisches Ziel und dazu nötige Gestaltungsbereiche für ihre Aktivitäten definieren.

Eine geeignete Methode dazu sind Reifegradmodelle, da sie ermöglichen, den Status quo zu ermitteln (Becker et al. 2009) und gleichzeitig einen Entwicklungspfad zu einem möglichen Zielzustand aufzeigen (Pöppelbuß/Röglinger 2011; Raber 2013). Damit bieten sie eine Struktur für eine wohlinformierte strategische Unternehmensführung und einen Bezugsrahmen für die Zielsetzung von Transformationsprozessen. Reifegradmodelle skizzieren eine schrittweise und systematische Verbesserung von Fähigkeiten, Prozessen oder Strukturen (Blondiau et al. 2013). Ein Reifegradmodell ist also ein Werkzeug für Manager, 
mit dessen Hilfe ein Bewusstsein für die relevanten Gestaltungsbereiche im Unternehmen geschaffen und ein fortlaufender Entwicklungsprozess strukturiert begleitet werden kann.

Im Bereich Information Systems (IS) existiert bereits eine Vielzahl an Reifegradmodellen. Die Analyse zeigt aber, dass sich bestehende Reifegradmodelle häufig auf einen eng definierten Bereich beziehen (Mettler 2010; Cleven et al. 2014; Neff et al. 2014) und nur wenige Modelle mehrere Unternehmensbereiche berücksichtigen (Albu/Panzar 2010). Zudem ist es - gerade bei Veränderungsprozessen, die die gesamte Organisation betreffen - wichtig, dass die Mitarbeiterperspektive, die Unternehmenskultur und die Zusammenarbeit nicht vernachlässigt werden, da Veränderungen immer auch ein Prozess der Wissensentwicklung und -weitergabe sind (Margherita/Petti 2010; Zephir et al. 2011; Walton 2014). Einige bestehende Reifegradmodelle von Unternehmensberatungen versuchen den Fokus breiter zu legen (Westerman et al. 2012; Sonntag/Müller 2013; BearingPoint Ltd. 2014; Land 2014; Lloyds Bank 2014; Steimel et al. 2014;). Allerdings fehlt bei diesen Modellen häufig die rigorose Dokumentation des methodischen Ansatzes, wie er bei Becker et al. (2009) oder De Bruin et al. (2005) gefordert wird.

Daher möchten wir in dieser Studie ermitteln, welche Bereiche eines Unternehmens im Zuge einer digitalen Transformation der Organisation neu gestaltet werden müssen und ein Reifegradmodell entwickeln, welches zur Analyse der eigenen Transformationsaktivitäten und als Strukturierungshilfe für die Gestaltung des Digitalisierungsprozesses dienen soll.

\section{Konzeptionelle Grundlage}

In unserer Studie folgten wir einem explorativen Ansatz, in dem wir uns zwar an bestehenden Modellen orientierten, aber bewusst keine theoretische Grundlage gewählt haben, um auch neue Konzepte zu entdecken und in das Modell einzuschliessen.

Als konzeptionelle Grundlage zur Betrachtung der digitalen Transformation eignen sich mehrere Ansätze. Die Perspektive des „Organizational Learning“ kann angewendet werden, um zu verstehen, wie neues Wissen im Unternehmen generiert und verbreitet wird (Argyris/Schön 1996; Lyytinen et al. 2010). Andere Studien basieren auf dem RessourceBased-View (RBV) (Barney 1991) oder Dynamic Capabilities (Teece et al. 1997; Eisenhardt/ Martin 2000), die davon ausgehen, dass wertvolle, seltene, nicht imitierbare und unersetzbare Ressourcen und Fähigkeiten zum Unternehmenserfolg beitragen.

Diese genannten Ansätze fokussieren sich auf interne Fähigkeiten und Abläufe im Unternehmen und sparen die detaillierte Betrachtung von Customer Experience, Produkten und Dienstleistungen aus.

Das Technology-Organization-Environment (TOE) Framework (Tornatzky et al. 1990) berücksichtigt den Kontext von Transformations- und Innovationsentscheidungen in den drei Dimensionen Technologie, Organisation und Marktumfeld. Dieses Framework eignet sich dazu, Treiber zu identifizieren, aber weniger, um den Transformationsprozess des Unternehmens zu betrachten.

Die Disziplin Business Engineering hat die methodische Transformation von Unternehmen des Industriezeitalters in das Informationszeitalter zum Ziel (Österle 1995; Baumöl 2008). Die St.Galler Business-Engineering-Landkarte erlaubt eine systematische Betrachtung der organisationalen Transformation im digitalen Zeitalter. Vier Eigenschaften des Business Engineering waren ausschlaggebend für die Auswahl als Ordnungsrahmen im Rahmen unserer Studie: Erstens fokussiert sich der Ansatz auf Veränderungs- und Erneue- 
rungsprozesse von Unternehmen im Gegensatz zu inkrementellen Weiterentwicklungen im Rahmen des Tagesgeschäfts (Winter 2011). Zweitens ist Business Engineering sowohl als Verständnis- als auch als Gestaltungsrahmen zu verstehen, welcher die Analyse von Handlungsfeldern und die Ausarbeitung von Massnahmen gleichermassen unterstützt (Winter 2011). Drittens erlaubt Business Engineering eine ganzheitliche Betrachtungsweise von komplexen, digitalen Transformationsprozessen, da das Modell auf vernetzte Geschäftsarchitekturen abzielt und neben der fachlichen Dimension die Bedeutung von Informationstechnologien in hohem Masse berücksichtigt (Österle/Winter 2011). Viertens hat die Business-Engineering-Landkarte einen hohen praktischen Anwendungswert, da sie im Dialog mit Praktikern und Wissenschaftlern kontinuierlich weiterentwickelt wird.

Im Rahmen dieser Studie dient uns die Business-Engineering-Landkarte als Ordnungsrahmen, innerhalb dessen wir die Dimensionen herausarbeiten, welche innerhalb der digitalen Transformation wichtige Handlungsfelder darstellen.

Das Kernmodell umfasst die Bereiche Strategie, Prozesse und Informationstechnologie (Österle 1995; Österle/Winter 2003). Das neue „St.Galler House of Digital Business“ berücksichtigt neben dem Bereich „Führungsaufgaben“ auch die Dimensionen „Produktund Leistungssicht“ sowie „User-, Use-, Utility-Centricity“ als Dach des Modells, um die besondere Bedeutung der veränderten Kundenerwartungen in der neuen digitalen Realität hervorzuheben (Brenner et al. 2014; Leimeister et al. 2014).

\section{Methodisches Vorgehen}

Zur Erstellung des Digital Maturity Model dienten die von Becker et al. (2009) und De Bruin et al. (2005) beschriebenen Hinweise zur Gestaltung von Reifegradmodellen als Grundlage für unsere Vorgehensweise.

\subsection{Literatur Review}

Zum Design der Dimensionen, der Reifekriterien und der zugehörigen Messinstrumente wurden insgesamt 70 wissenschaftliche Publikationen zum Thema Digital Business Transformation sowie 16 bestehende vergleichbare Reifegrad-Assessments analysiert. Die wissenschaftlichen Publikationen wurden über eine strukturierte Literatursuche in den Datenbanken EBSCOhost, AISeL, Springer Link, Sciencedirect und Emerald Insight identifiziert, so dass gleichermassen Management- und IS-Publikationen berücksichtigt wurden. In den Datenbanken wurde mit Kombinationen der Suchbegriffe „business transformation“, „enterprise transformation“, „organi?ational transformation“, „organi?ational change“, „digital transformation“, „success factor“ und „maturity model“ gesucht.

Die Suche in den Datenbanken war eingeschränkt auf wissenschaftliche Publikationen seit dem Jahr 2005. Die Relevanz der gefundenen Publikationen wurde anhand von Titel und Abstract eingeschätzt. Ausgeschlossen wurden Publikationen, in denen die Digitalisierung ohne Berücksichtigung der Auswirkungen auf den Unternehmenskontext untersucht wurde, die sich auf einen sehr spezifischen Kontext beschränkten (z.B. Digital Government) sowie Publikationen, bei denen explizit spezifische kulturelle Gegebenheiten untersucht wurden. 


\subsection{Experteninterviews}

Die Erkenntnisse aus der Literaturanalyse wurden um die Praxissicht durch Interviews und Fokusgruppen erweitert. An die Teilnehmer aus der Praxis wurden folgende Anforderungen gestellt:

- Mindestens 5 Jahre Berufserfahrung.

- Entscheidungsträger, die das Thema „Digitale Transformation“ in ihren Unternehmen oder als Dienstleister für Anwenderunternehmen operativ verantworten.

- Zusammensetzung aus verschiedenen Branchen.

Zunächst wurden sieben telefonische Experteninterviews anhand eines semistrukturierten Leitfadens durchgeführt, die jeweils ca. 45 Minuten dauerten. Die Interviews wurden aufgezeichnet und anschliessend transkribiert.

Zunächst wurden die Teilnehmer zu ihrem beruflichen Hintergrund und zu ihrer Position befragt, dann zu den Herausforderungen der digitalen Transformation in ihrem Unternehmen und zu Aktivitäten und Erfolgsfaktoren in den Bereichen Strategie, Prozesse, Technologie, Produktinnovation und Kultur und Führung.

\subsection{Codierung}

Sowohl die in der Literaturschau identifizierten Publikationen als auch die Transkripte der Interviews wurden codiert. Basis für das Coding-Schema waren die Dimensionen des „St.Galler House of Digital Business“ (Leimeister et al. 2014) - „Strategie“, „Prozesse“, „Technologie“, „Leistungssicht“, „User“ und „Kultur“. Mit diesem Schema als Gerüst wurde offen codiert, z.B. „Strategie - Flexibilität“. Die entstandenen Codes wurden danach thematisch zusammengefasst, z.B. im Bereich Kultur wurden die Codes „KnowHow“, „Skills“, „Offenheit“, „Digitale Expertise“ zum Cluster „Digitale Expertise“ zusammengefasst, was in einer weiteren Iteration in „Digitale Affinität“ umbenannt wurde, um nicht nur Spezialwissen, sondern auch Breitenwissen damit abzudecken. Auf diese Weise wurden aus den zusammengefassten Codes die Dimensionen und danach die Reifekriterien des Modells konsolidiert. Für den Codierungsprozess wurde die Software Atlas.ti verwendet.

\subsection{Fokusgruppen}

Die im Codierungsprozess identifizierten Dimensionen wurden in einer Fokusgruppe mit elf Teilnehmern während drei Stunden evaluiert. Die Fokusgruppe wurde anhand eines Leitfadens moderiert und von einem separaten Protokollanten dokumentiert. Die Zwischenergebnisse waren an einer Pinnwand für alle sichtbar.

Zunächst wurden die Dimensionen präsentiert und ein freies Brainstorming zu relevanten Fähigkeiten und Aktivitäten in diesen Bereichen durchgeführt. Danach wurden die aus der Literatur erarbeiteten Kriterien ergänzt und die Unterschiede diskutiert. Anschliessend wurde in eine offene Diskussion übergeleitet.

Im Anschluss an die Fokusgruppe wurde das Modell anhand des Feedbacks überarbeitet und mit der Erstellung der Indikatoren für den Kriterienkatalog begonnen. Im nächsten Schritt waren die Teilnehmer der Fokusgruppe aufgefordert, über ein gemeinsam bearbeitbares Online-Dokument (GoogleDocs) die vorgeschlagenen Indikatoren zu bearbeiten, zu kritisieren oder zu ergänzen. 
Das Ergebnis dieser digital geführten Diskussion wurde in einer zweiten Fokusgruppe mit sechs Teilnehmern in einem vierstündigen Workshop besprochen. Dazu wurde der erarbeitete Indikatoren-Pool präsentiert und für jede Dimension des Modells von den Teilnehmern anhand der Evaluationskriterien „Relevanz“, „Vollständigkeit“ und „Verständlichkeit" beurteilt. Im Anschluss an die Fokusgruppe wurde auf Basis des Feedbacks der finale Kriterienkatalog mit 59 Indikatoren $^{1}$ definiert.

\subsection{Erstellung des Kriterienkatalogs}

Für die Erstellung des Kriterienkatalogs wurden für alle Kriterien bestehende Messinstrumente recherchiert und wo möglich adaptiert.

\begin{tabular}{|l|l|}
\hline Reifekriterium & Indikatoren adaptiert von: \\
\hline Unternehmerische Agilität & $\begin{array}{l}\text { (Chakravarty et al. 2013) } \\
\text { Entrepreneurial Agility }\end{array}$ \\
\hline Flexibles Arbeiten & $\begin{array}{l}\text { (Weichbrodt et al. 2014) } \\
\text { FlexWork Phasenmodell. Ausprägungen der Stufe 5 (Netz- } \\
\text { werk-Unternehmen) dienten als Vorlage für die Indikatoren. }\end{array}$ \\
\hline Fehlerkultur & $\begin{array}{l}\text { (van Dyck et al. } 2005) \\
\text { Error Management Culture }\end{array}$ \\
\hline Performance Measurement & $\begin{array}{l}\text { (Quadgras et al. 2014) } \\
\text { Action-oriented Assessment }\end{array}$ \\
\hline
\end{tabular}

Tabelle 1: Adaptierte Messinstrumente

Tabelle 1 zeigt eine Übersicht über die adaptierten Messinstrumente. Für die übrigen Kriterien wurde analog zum von DeVellis (2003) vorgeschlagenem Vorgehen zunächst ein Indikatoren-Pool entwickelt und daraus der erste Draft des Kriterienkatalogs entwickelt. Die vorgeschlagenen Indikatoren wurden in der zweiten Fokusgruppe als relevant, vollständig und verständlich bewertet. Teilweise wurden die Listen gekürzt, um den Kriterienkatalog übersichtlich zu halten. Der finale Kriterienkatalog wurde ausserdem in einem Pretest mit drei Teilnehmern hinsichtlich Verständlichkeit, Angemessenheit und Konsistenz evaluiert.

\section{Digital Maturity Model}

Die in der Literatur und in den Experteninterviews identifizierten Faktoren wurden in Dimensionen zusammengefasst. Für jede Dimension wurden entsprechende Reifekriterien definiert, welche die Handlungsfelder beschreiben. Handlungsfelder ergeben sich aus identifizierten Aktivitäten, durch die im Zusammenhang mit der Digitalisierung Mehrwerte geschaffen oder neue Möglichkeiten eröffnet werden könnten, aber auch Fähigkeiten und Voraussetzungen in einem Unternehmen. Aktivitäten in diesen Feldern zeigen die Durchdringung der digitalen Reife.

Als finale Dimensionen wurden „Customer Experience“, „Produktinnovation“, „Strategie“, „Organisation“, „Prozessdigitalisierung“, „Zusammenarbeit“, „ICT-Betrieb \& -Ent-

1 Siehe Anhang A. 
wicklung“, „Kultur \& Expertise“ und „Transformationsmanagement“ für das Digital Maturity Model identifiziert (siehe Abbildung 1).

\section{Customer}

Experience

\section{Kultur \& Expertise}

\section{Strategie}

Organisation

Transformationsmanagement

\section{Prozess- \\ digitalisierung}

Abbildung 1: Digital Maturity Model

\begin{tabular}{|c|c|c|c|}
\hline Dimension / Reifekriterien & $\begin{array}{l}\text { Literatur-Re- } \\
\text { view }\end{array}$ & $\begin{array}{l}\text { Experten-In- } \\
\text { terviews }\end{array}$ & $\begin{array}{l}\text { Fokusgruppe } \\
1\end{array}$ \\
\hline \multicolumn{4}{|l|}{ 1. Customer Experience } \\
\hline Analytics & $(\checkmark)$ & $\checkmark$ & $\checkmark$ \\
\hline Cross-Channel Experience & $(0)$ & $\checkmark$ & $\checkmark$ \\
\hline \multicolumn{4}{|l|}{ 2. Produktinnovation } \\
\hline Geschäftsfelderweiterung & $\checkmark$ & $\checkmark$ & $\checkmark$ \\
\hline Innovationsgrad & $\checkmark$ & $(\checkmark)$ & $\checkmark$ \\
\hline Digitale Kundenintegration & $(\sqrt{ })$ & $\checkmark$ & $\checkmark$ \\
\hline \multicolumn{4}{|l|}{ 3. Strategie } \\
\hline Strategische Innovation & $(\checkmark)$ & $\checkmark$ & $\checkmark$ \\
\hline Digitales Commitment & $\checkmark$ & $(\checkmark)$ & $\checkmark$ \\
\hline \multicolumn{4}{|l|}{ 4. Organisation } \\
\hline Digitale Teamaufstellung & $(\checkmark)$ & $\checkmark$ & $\checkmark$ \\
\hline Unternehmerische Agilität & $\checkmark$ & $\checkmark$ & $\checkmark$ \\
\hline Partnernetzwerk & $\checkmark$ & $\checkmark$ & $\checkmark$ \\
\hline \multicolumn{4}{|l|}{ 5. Prozessdigitalisierung } \\
\hline Touchpoint Management & $(\checkmark)$ & $(\checkmark)$ & $\checkmark$ \\
\hline Automatisierung & $\checkmark$ & $\checkmark$ & $\checkmark$ \\
\hline Digitale Marketingkommunikation & $(0)$ & $(\checkmark)$ & $\checkmark$ \\
\hline
\end{tabular}




\begin{tabular}{|l|c|c|c|}
\hline Dimension / Reifekriterien & $\begin{array}{l}\text { Literatur-Re- } \\
\text { view }\end{array}$ & $\begin{array}{l}\text { Experten-In- } \\
\text { terviews }\end{array}$ & $\begin{array}{l}\text { Fokusgruppe } \\
1\end{array}$ \\
\hline 6. Zusammenarbeit & & & \\
\hline Kollaboration & $\checkmark$ & $\checkmark$ & $\checkmark$ \\
\hline Wissensmanagement & $\checkmark$ & $(\checkmark)$ & $\checkmark$ \\
\hline Flexibles Arbeiten & & $\checkmark$ & $\checkmark$ \\
\hline 7. ICT-Betrieb und Entwicklung & $(\checkmark)$ & $\checkmark$ & $\checkmark$ \\
\hline Agile Projektabwicklung & $(\checkmark)$ & $\checkmark$ & $\checkmark$ \\
\hline Integrierte Architektur & $\checkmark$ & $\checkmark$ & $\checkmark$ \\
\hline IT-Expertise & & & \\
\hline 8. Kultur und Expertise & $(0)$ & $\checkmark$ & $\checkmark$ \\
\hline Digitale Affinität & $(0)$ & $\checkmark$ & $\checkmark$ \\
\hline Risikobereitschaft & & & \\
\hline Fehlerkultur & $\checkmark$ & $\checkmark$ & $\checkmark$ \\
\hline 9. Transformationsmanagement & $(\checkmark)$ & $\checkmark$ & $\checkmark$ \\
\hline Governance & $\checkmark$ & $\checkmark$ & $\checkmark$ \\
\hline Management-Unterstützung & & $\checkmark$ \\
\hline Performance Management & & $\checkmark$ & \\
\hline$\checkmark=$ häufige Nennung & $\checkmark$ & & \\
\hline
\end{tabular}

Tabelle 2: Identifizierte Dimensionen und Reifekriterien in den einzelnen Schritten der Studie

\subsection{Customer Experience}

Die Kundenbedürfnisse und -erwartungen haben sich durch die Digitalisierung und die Durchdringung von Technologien im Alltag verändert (Brenner et al. 2014). Dies wurde insbesondere auch durch die Interviews mit Experten aus der Praxis deutlich, die dies als einen zentralen Treiber der digitalen Transformation nannten:

"Ich bin der Überzeugung, dass die Kunden nicht mehr auf alte Mechanismen anspringen [...] Die Kernkundenbasis wächst immer mehr in die Digital Natives rein und die agieren anders, informieren sich anders, schliessen anders ab." (Director Online, Telekommunikation)

Ein wichtiges Handlungsfeld für Unternehmen ist daher die Fähigkeit, die Nutzerbedürfnisse zu verstehen (Liang/Tanniru 2006; Iivari/Iivari 2011) und ihre Angebote auf dieses veränderte Verhalten der Kunden hin auszurichten.

Als erstes Reifekriterium (Analytics) für Unternehmen wurde die Fähigkeit definiert, über digitale Möglichkeiten Kundendaten aus verschiedenen Quellen zusammenzuführen, um Wissen über den Kunden zu generieren und zukünftiges Verhalten zu antizipieren (Westerman et al. 2011; PwC 2013). 
Das zweite Reifekriterium (Cross-Channel Experience) ist die Fähigkeit, die digitalen Angebote auf die Kundenbedürfnisse auszurichten (Liang/Tanniru 2006; Chaffey 2010; Westerman et al. 2011; Gray et al. 2013) und mit den Kunden nahtlos über verschiedene Kanäle zu interagieren.

\subsection{Produktinnovation}

Der hohe Durchdringungsgrad von digitalen Technologien, wie z.B. mobile Endgeräte, oder die Verfügbarkeit von kontextsensitiven Informationen ermöglichen Unternehmen ihr physisches Leistungsangebot mit digitalen Möglichkeiten zu erneuern (Yoo et al. 2010).

Als erstes Reifekriterium (Geschäftsfelderweiterung) wurde die Bereitschaft definiert, dass ein Unternehmen bestehende Produkte und Services mit neuen digitalen Lösungen ergänzt und dadurch relevante digitale Geschäftsfelder oder Ertragsströme (Berman 2012) erschliesst.

Damit verbunden ist die Fähigkeit zur Innovation und die Entwicklung und Umsetzung von innovativen Ideen (Innovationsgrad) (Berman 2012; Henfridsson/Yoo 2014). In den Diskussionen mit Praktikern wurde vor allem die Notwendigkeit hervorgehoben, dass Rahmenbedingungen für ein innovatives Umfeld unter den Mitarbeitern geschaffen werden:

„Wichtig ist, dass Unternehmen eine Ideenplattform abseits von Hierarchien schaffen. Wird das überhaupt im Unternehmen zugelassen?" (Managing Director, Verwaltung / Schulen)

In einer digitalen Welt wird Innovation in besonderem Masse durch die Einbindung von Kunden oder eine verstärkte Vernetzung der eigenen Mitarbeiter ermöglicht. Offene Strukturen und der Einbezug von Lead Usern fördern die erfolgreiche Entwicklung von innovativen Ideen (Leimeister et al. 2009).

„Wir haben [...] Focus Days, da kann man an einem Thema arbeiten, das einen interessiert, aber das keinen Projektzusammenhang hat. Da kommen aus allen Bereichen Ideen und [...] nach zwei Tagen werden die Ergebnisse präsentiert. Das ist ein kleines Innovation Lab. " (Leiter Digital Business Applications, Versicherung)

Daher wurde die „Digitale Kundenintegration“ als drittes Reifekriterium für die Dimension Produktinnovation übernommen.

\subsection{Strategie}

Die strategische Planung digitaler Innovation ist nicht allein Gegenstand in der IT-Strategie, sondern Inhalt einer Fachbereiche und IT-Abteilung übergreifenden digitalen Geschäftsstrategie (Bharadwaj et al. 2013; Drnevich/Croson 2013).

Als wichtige Voraussetzung für die digitale Transformation wurde „Strategische Innovation" identifiziert. Dies bedeutet, dass Innovation durch digitale Technologien eine grosse Rolle in der Strategie spielt und aktiv vorangetrieben wird (Yoo et al. 2010). Dies wurde auch durch Aussagen aus den Experteninterviews gestützt:

"Wir investieren jährlich 1,3 Mrd US Dollar in Weiterentwicklung, es gibt nicht viele Unternehmen, die das stemmen können, [...] Es ist wichtig, dass wir die Industrie da vorantreiben und diese disruptiven Elemente entwickeln." (Managing Director, Banken) 
Weiterhin wurde von den Experten die zentrale Rolle der IT und digitaler Themen in der Unternehmensstrategie hervorgehoben, was auch durch verschiedene wissenschaftliche Arbeiten gestützt wird (Chen et al. 2010; Leidner et al. 2011; Bharadwaj et al. 2013; Mithas et al. 2013b). „Digitales Commitment“" wurde als weiteres Reifekriterium identifiziert.

\subsection{Organisation}

Die digitale Transformation erfordert auch eine Neuaufstellung in der Organisationsstruktur, um eine grössere Agilität und Flexibilität in der Umsetzung von digitalen Projekten zu gewährleisten.

Das erste identifizierte Reifekriterium ist eine digitale Teamaufstellung. Organisationale Silos sind aufgelöst und digitale Kompetenzen sind in allen Unternehmensbereichen vorhanden. Eine digitale Teamaufstellung zeigt sich beispielsweise darin, dass Fachabteilungen enger mit IT-Abteilungen an digitalen Projekten zusammenarbeiten ( $Q u$ et al. 2010; Hansen et al. 2011). Von den befragten Praktikern wurden in diesem Zusammenhang auch eine stärkere dezentrale Verteilung von Entscheidungskompetenzen und Budget erwähnt:

„Digital Business wird auf einen Nucleus reduziert, das die gesamte Digital Transformation auf strategischer Ebene stenert. Die einzelnen Ressorts haben Kapazitäten und Expertise und dezentrales Budget. " (Leiter Digitale Transformation, Versicherungen).

Die hohe Dynamik der digitalen Transformation erfordert darüber hinaus eine unternehmerische Agilität. Das Unternehmen besitzt die Fähigkeit, flexibel auf wechselnde Einflüsse von aussen zu reagieren (Sambamurthy et al. 2003; Ganguly et al. 2009; Chakravarty et al. 2013).

Im Zusammenhang mit einer höheren Flexibilität steigt die Notwendigkeit eines Partnernetzwerks. Für fehlende Fähigkeiten zur Entwicklung von externen Innovationen oder zum Überbrücken von kurzfristigen Engpässen kann ein Unternehmen auf ein Netzwerk aus Spezialdienstleistern zurückgreifen (Grover/Kobli 2012; Frankenberger et al. 2013).

\subsection{Prozessdigitalisierung}

Im Bereich Prozesse werden digitale Technologien eingesetzt, um interne Prozesse sowie externe Prozesse effizienter zu gestalten und wenn möglich zu automatisieren. Dies ist stark von den Voraussetzungen der jeweiligen Branche abhängig. Für eine branchenübergreifende Betrachtung wurden die folgenden Handlungsfelder identifiziert:

In der externen Kommunikation mit digitalen Kunden, über mehrere Plattformen hinweg, ist es wichtig, dass digitale und mobile Kanäle mit internen Prozessen nahtlos integriert sind (Touchpoint Management) (Chaffey 2010). Dies geht über die konsistente Gestaltung der Interfaces hinaus, weswegen dieses Kriterium auf der Prozessebene verortet ist.

Damit verbunden ist die Fähigkeit, digitale Massnahmen in der Kommunikation auf Basis von Daten zu steuern und Entscheidungen datenbasiert zu treffen (Digitale Marketingkommunikation).

Ein weiteres Handlungsfeld ist die Automatisierung. Ein Unternehmen schöpft digitale Möglichkeiten aus, um Kernprozesse und Routineprozesse zu vereinfachen und effizienter zu gestalten (Bartel et al. 2005). 
„Wir arbeiten kontinuierlich daran, dass wir Prozesse zuerst vereinfachen und dann versuchen, zu automatisieren. Wir streben das vor allem in Prozessen an, wo ein Mensch keinen Mehrwert in Richtung Kunde stiften kann und da spielen digitale Möglichkeiten eine grosse Rolle." (Leiter Digital Business Applications, Versicherungen).

\subsection{Zusammenarbeit}

Digitale Technologien können innerhalb des Unternehmens die Kommunikation und Kollaboration der Mitarbeiter verbessern. Auf diese Weise hat die digitale Transformation einen Einfluss auf die Mechanismen in der Zusammenarbeit.

Im Bereich des Wissensmanagements erlauben digitale Technologien in besonderem Masse, Mitarbeiter aus verschiedenen Unternehmensbereichen miteinander zu vernetzen und so Wissensprozesse zu stärken (Sambamurthy et al. 2003). Dies kann auch dazu genutzt werden, um Innovationsprozesse zu öffnen und externe Ressourcen besser einzubinden (Enkel et al. 2011).

Ein weiteres Handlungsfeld ist der Einsatz von digitalen Technologien im Bereich Kollaboration von Teams, z.B. zur Kommunikation und Weitergabe von Informationen. Dies ist der wachsenden Vernetzung von Teams, z.B. auf globaler Ebene, geschuldet (Smith/McKeen 2011).

In diesem Zusammenhang spielt auch die Möglichkeit zu flexiblem Arbeiten eine wichtige Rolle. Durch den Einsatz von digitalen Technologien kann das Unternehmen wechselnde Arbeitsorte und mobiles Arbeiten unterstützen.

Die befragten Praktiker bestätigten dies als wichtiges Handlungsfeld, v.a. in der Vernetzung von Mitarbeitern ohne festen Arbeitsort:

„Der Trend, den wir beobachten, geht mehr aus dem Büro raus zum Arbeiter im Feldder wird vernetzter, und wir können so Projektmitarbeitende verbinden. Sämtliche Mitarbeitende sollen bis nächstes Jahr mit Smartphones ausgestattet werden." (Leiter Mobile IT, Logistik \& Transport)

\subsection{ICT-Betrieb und Entwicklung}

Auf Ebene der ICT-Systeme werden die Grundlagen für neue digitale Produkte, Dienstleistungen, Kommunikation und Transaktionen gelegt. Hier wurden drei relevante Handlungsfelder identifiziert:

Digitale Projekte werden zunehmend mit agilen Methodiken abgewickelt. So können Unternehmen digitale Lösungen schnell in Betrieb nehmen und anpassen. Für Unternehmen bedeutet dies aber auch eine Anpassung der Steuerungsmechanismen und Strukturen in der Projektabwicklung (Aaen et al. 2007; Wiklund et al. 2013). Auch in den Experteninterviews wurden agile Methoden als entscheidender Erfolgsfaktor in digitalen Projekten genannt:

„Wir arbeiten mit agilen Projektabwicklungsmethoden, wo wir in drei bis vier Wochen ein Delivery machen, ein Produkt liefern können, wo wir schnell Feedback bekommen und wo wir schnell Fehler machen und diese auch schnell korrigieren können." (Leiter Digital Business Applications, Versicherungen)

Um neue digitale Lösungen schnell an bestehende Systeme anzubinden, ist eine skalierbare, integrierte Architektur nötig. Hier spielen Investitionen in die Qualität der IT-Infrastruktur 
und die Agilität der Backend-Systeme eine wichtige Rolle, um eine entsprechende Flexibilität zu gewährleisten (Sambamurthy et al. 2003; Liu et al. 2011; Chakravarty et al. 2013).

Darüber hinaus ist ein weiterer wichtiger Handlungsbereich, in der internen IT die Expertise in den für das Unternehmen definierten Schlüsseltechnologien aufzubauen (Bharadwaj 2000; Bi et al. 2011; Chakravarty et al. 2013), um die Fachabteilungen entsprechend beraten zu können oder ggf. externe Dienstleister zu führen.

\subsection{Kultur und Expertise}

In der Literaturanalyse von Publikationen im Bereich digitale Transformation wurden kulturelle Voraussetzungen nur in geringem Masse genannt (Philip/McKeown 2004). In den Experteninterviews und der ersten Fokusgruppe wurde dagegen die hohe Bedeutung von kulturellen Rahmenbedingungen in der Organisation hervorgehoben.

Damit die Mitarbeiter die Transformation eines Unternehmens mittragen, ist wichtig, dass diese eine hohe digitale Affinität aufweisen. Dies bedeutet, dass sie nicht nur das für ihre Rolle relevante Spezialwissen, sondern auch ein „Breitenwissen“ zu digitalen Technologien aufweisen und motiviert sind, diese Anwendungen in ihrem Arbeitsalltag zu verwenden (Harris et al. 2012).

Die Teilnehmer aus der Praxis hoben als weitere wichtige kulturelle Voraussetzungen Risikobereitschaft und eine offene Feblerkultur hervor. Gerade bei sehr innovativen Projekten muss das Unternehmen bereit sein, Risiken einzugehen und bestehende Vorgehensweisen oder Produkte aufzugeben. Damit verbunden ist die Fähigkeit, Fehler offen zu kommunizieren und als Weiterentwicklungsmöglichkeit für das Unternehmen zu begreifen.

„Wie kann ich denn ein "Gamechanging Marketplayer» sein, wenn ich immer die sichere Variante wäble? Ich muss [...] eine Kultur haben, die Lust auf Risiken macht und Feblschläge zelebriert und Wissen daraus generiert. " (Director Marketing, Telekommunikation)

\subsection{Transformationsmanagement}

Die digitale Transformation eines Unternehmens ist nicht allein Sache der IT, sondern ein bereichsübergreifendes Veränderungsprojekt. Daher ist eine entsprechende Planung und Steuerung dieses Prozesses nötig.

„Es braucht eine neue Organisationsstruktur, neue Governance. Da reicht es nicht eine Online Unit aufzubauen, da müssen alle an einem Strang ziehen, die brauchen klare Ziele und Guidelines. “ (Leiter Digital Business Transformation, Versicherungen)

Die Dimension Transformationsmanagement wird durch drei Handlungsfelder definiert:

Erstens die Definition einer neuen Governance mit definierten Rollen und Verantwortlichkeiten für alle mit der digitalen Transformation des Unternehmens verbundenen Aktivitäten (Otto 2010; Grover/Kohli 2012; Chatterjee/Ravichandran 2013; Tiwana et al. 2013).

Zweitens ist die Unterstützung des Top Managements entscheidend, das die digitale Transformation des Unternehmens vorantreibt (Jahani et al. 2010) und Ressourcen bereitstellt. 
Der dritte Gestaltungsbereich ist das Performance Measurement der Transformation, also die Definition und regelmässige Überprüfung von Zielwerten für entsprechende Aktivitäten (Janssen et al. 2011; Buchwald et al. 2014; Labusch et al. 2014).

\section{Diskussion der Ergebnisse und der Methodik}

\subsection{Kritische Würdigung der Anwendung von Reifegradmodellen}

Reifegradmodelle sind in der Wirtschaftsinformatik ein häufiges Werkzeug für die Unternehmensentwicklung (Becker et al. 2010). In der aktuellen Literatur findet sich aber auch Kritik an der Entwicklungsmethodik und der Anwendung von Reifegradmodellen. In einer Studie von Mullaly (2014) wird die Frage gestellt, ob Reifegradmodelle tatsächlich eine Vorlage für Projekte darstellen können und damit ein strategischer Vorteil für das Unternehmen zu erzielen ist. Wir denken, dass dies im Sinne eines Rahmenwerks durchaus möglich ist, allerdings sind konkrete Handlungsanweisungen von einem solchen Modell nicht abzuleiten.

Dies ist vor allem dem Umstand geschuldet, dass das Konzept von „Reife“ schwer zu definieren ist. Reifegradmodelle basieren häufig auf der Annahme, dass der Fortschritt hin zu einem Zielzustand ein linearer Prozess ist und ein vermeintlich reifes Unternehmen auch unternehmerisch besser aufgestellt ist als die Wettbewerber. Die meisten Reifegradmodelle bieten lediglich ein generisches Bezugssystem an, das sich nicht auf alle Projekte innerhalb eines Unternehmens anwenden lässt, da dies bedeuten würde, dass auch sehr individuelle Projekte auf die gleiche Art und Weise geführt werden müssten. Reifegradmodelle sind daher nicht geeignet, um sehr spezifische Probleme zu lösen (Cleven et al. 2014; Mullaly 2014; Pries-Heje/Baskerville 2010).

Damit Reifegradmodelle ein nützliches Instrument zur Verbesserung von Transformationsprozessen werden können, müssen sie nicht nur den aktuellen Status des Unternehmens untersuchen (deskriptiv), sondern auch konkrete Schritte aufzeigen, wie die nächste Reifestufe erreicht werden kann, so dass Entscheidungsträger einen Entwicklungspfad definieren können (präskriptiv) (Jahani et al. 2010). In einer Analyse von 10 Reifegradmodellen zeigten Röglinger et al. (2012), dass den Designprinzipien für präskriptive Verwendungen selten entsprochen wird. Daher sind diese Modelle von begrenztem Wert für Unternehmen.

In dieser Studie wurde ein neues Reifegradmodell zur Evaluierung der digitalen Reife von Unternehmen entwickelt. Auch in unserer Untersuchung zeigte sich, dass ein Reifegradmodell in seiner deskriptiven Funktion gut geeignet ist, um den aktuellen Fähigkeitsstand von Unternehmen abzubilden und sich auf diese Weise einzuschätzen. Präskriptive Funktionen, gerade bei einem so komplexen Feld und einem weit gefassten Branchenfokus, sind allerdings in ein solches Modell schwierig zu integrieren. Daher würden wir den präskriptiven Beitrag von Reifegradmodellen ebenfalls eher gering einschätzen. So sollte das Digital Maturity Model nicht als normative Handlungsanweisung verstanden werden, sondern als beschreibendes Element, das durch die Beschäftigung mit den verschiedenen Themengebieten der digitalen Transformation Hilfestellung bei der Gestaltung der individuellen Entwicklungspfade im konkreten situativen Kontext eines Unternehmen gibt. 


\subsection{Praktischer Beitrag des Digital Maturity Model}

Der Kriterienkatalog des Digital Maturity Model stellt für Entscheidungsträger ein Werkzeug in der Analysephase des Managementkreislaufs dar und liefert einen konkreten Beitrag als Standortbestimmung und als gemeinsame Gesprächsgrundlage („Boundary Object").

Zum einen dient die Beschäftigung mit dem Kriterienkatalog der systematischen Analyse ihrer Veränderungsaktivitäten sowie allfälliger Schwachstellen in ihrem Transformationsprozess. Besonders hervorzuheben ist in diesem Zusammenhang das Aufdecken von Wahrnehmungsdifferenzen innerhalb der Organisation. Wird der Kriterienkatalog von mehreren Personen aus demselben Unternehmen angewendet, gibt dies Aufschluss, welche Bereiche unterschiedlich eingeschätzt werden. Das Digital Maturity Model und die dazugehörige Umfrage dienen also als Standortbestimmung für die digitale Transformation eines Unternehmens.

Zweitens entsteht durch die Reflexion der Situation des Unternehmens ein Verständnis für die unterschiedlichen Auswirkungen der digitalen Transformation. Das Modell und die Beschäftigung mit dem Kriterienkatalog kann also als Grundlage für ein gemeinsames Verständnis („Boundary Object“) der digitalen Transformation dienen (Bechky 2003; Speel Jarzabkowski 2009) und einen entsprechenden Diskurs im Unternehmen anregen. Ein Teilnehmer der Umfrage formulierte dies so:

„Die Auseinandersetzung mit der Thematik hat uns in vielem, was wir in der Vergangenheit getan haben, bestärkt und hat dieser Perspektive der Unternehmensentwicklung ein Label gegeben." (Umfrageteilnehmer, Branche „Verwaltung und Schulen“)

\subsection{Weitere Forschungsfelder}

Der Fokus dieser Studie liegt in erster Linie auf dem praktischen Beitrag. Dennoch ergeben sich aus dieser Untersuchung weitere Forschungsfelder, um die Mechanismen und die strategische Planung der digitalen Transformation besser zu verstehen.

Zum einen ist die Anwendung und Evaluierung von Reifegradmodellen im Unternehmenskontext ein offenes Forschungsfeld. Der Rahmen dieser Studie ist auf die Entwicklung des Reifegradmodells beschränkt. Weitere Studien können evaluieren, welchen Beitrag, den dieses Modell in der Gestaltung von Transformationsaktivitäten im Unternehmen tatsächlich liefern und wie das Modell verbessert werden kann. Da die Literaturanalyse gezeigt hat, dass die präskriptive Funktion von Reifegradmodellen beschränkt ist, würden diese Erkenntnisse zur Verwendung von Reifegradmodellen im Unternehmenskontext einen wertvollen Beitrag in diesem Forschungsfeld liefern.

Ein weiteres vielversprechendes Forschungsfeld ist die Ergänzung der Reifegraduntersuchung mit Fallstudien. Hier könnte untersucht werden, wie Unternehmen die digitale Transformation als strategisches Programm aufsetzen, in welchen Bereichen priorisiert Aktivitäten geplant werden und welchen Erfolg Unternehmen daraus ziehen. Aus dem Wissen über verschiedene Digitalisierungsstrategien können zum einen unterschiedliche Transformationspfade, zum anderen Erfolgsfaktoren abgeleitet werden (Matt et al. 2015). 


\subsection{Limitationen der Untersuchung und Fazit}

Mit unserer Studie haben wir uns das Ziel gesetzt, die relevanten Gestaltungsbereiche zur Transformation von Unternehmen im digitalen Zeitalter in einem Reifegradmodell zu definieren.

Es ist anzumerken, dass eine finale Evaluierung des Reifegradmodells nötig ist, um dessen Beitrag als Instrument zur Unternehmensentwicklung im Arbeitsalltag konkret bewerten zu können. Da ein grosser Teil der Forschung sich lediglich auf das Design von Reifegradmodellen konzentriert und die Implementierung ausser Acht lässt, wäre ein wertvoller Beitrag zur Reifegradmodellforschung, wie Reifegradmodelle langfristig zum organisationalen Lernprozess beitragen können (Blondiau et al. 2013).

Trotz der genannten Limitation stellt das entwickelte Reifegradmodell eine wertvolle Erkenntnisbasis für weitere Forschungsfelder dar, die durch zusätzliche Studien erweitert werden kann.

Diese Studie liefert auf zwei Ebenen einen Beitrag. Zum einen wurde in einem transparent dokumentierten Prozess ein Reifegradmodell entwickelt, das die Einflussbereiche der digitalen Transformation ganzheitlich abbildet. Zum anderen bietet der entstandene Kriterienkatalog für Praktiker eine Möglichkeit, den eigenen Status quo zu analysieren und digitale Veränderungsprozesse strukturiert anzugehen. Auf diese Weise kann das Digital Maturity Model in Unternehmen konkret den Beitrag leisten, Initiativen zur digitalen Transformation zu bewerten und Anregungen geben, wie der digitale Wandel aktiv zu gestalten ist.

\section{Danksagung}

Unser Dank gilt Bramwell Kaltenrieder, Managing Partner der Strategieberatung Crosswalk, für die Unterstützung bei der Entwicklung und Durchführung der Studie sowie allen beteiligten Experten in den Interviews und Fokusgruppen.

\section{Literaturhinweise}

Aaen, I. et al. (2007). SPI Agility: How to Navigate Improvement Projects. Software Process Improvement and Practice, (12), 267-281.

Albu, E./Panzar, C. (2010). A new tool for assessing maturity alignment: The enterprise maturity matrix. Performance Improvement, 49(9), 35-47.

Argyris, C./Schön, D. A. (1996). Organizational learning II: theory, method, and practice. Reading Mass <etc.>: Addison-Wesley.

Barney, J. (1991). Firm Resources and Sustained Competitive Advantage. Journal of Management, 17(99), 120.

Bartel, A. P. et al. (2005). How Does Information Technology Really Affect Productivity? Plant-Level Comparisons of Product Innovation, Process Improvement and Worker Skills. Quarterly Journal of Economics, 122(4), 1721-1758.

Baumöl, U. (2008). Change Management in Organisationen. Situative Methodenkonstruktion für flexible Veränderungsprozesse. Wiesbaden: Gabler Verlag.

BearingPoint Ltd. (2014). Becoming a World Class Digital Organisation: Six best practices for digital transformation. Retrieved from http://www.bearingpoint.com/en-gb/adaptive-thinking/insight s/becoming-a-world-class-digital-organisation/. 
Bechky, B. A. (2003). Sharing Meaning Across Occupational Communities: The Transformation of Understanding on a Production Floor. Organization Science, 14(3), 312-330.

Becker, J. et al. (2009). Developing Maturity Models for IT Management. Business \& Information Systems Engineering, 1(3), 213-222.

Becker, J. et al. (2010). Maturity Models in IS Research. In ECIS 2010 Proceedings (Paper 42).

Beese, J. et al. (2015). On the Role of Complexity for Guiding Enterprise Transformations. In Aveiro, D. et al. (Eds.), Advances in Enterprise Engineering IX SE - 8 (Vol. 211, pp. 113-127). Springer International Publishing.

Berman, S. J. (2012). Digital transformation: opportunities to create new business models. Strategy \& Leadership, 40(2), 16-24.

Besson, P./Rowe, F. (2012). Strategizing information systems-enabled organizational transformation: A transdisciplinary review and new directions. The Journal of Strategic Information Systems, 21(2), 103-124.

Bharadwaj, A. (2000). A Resource-Based Perspective on Information Technology Capability and Firm Performance: An Empirical Investigation. MIS Quarterly, 24(1), 169-196.

Bharadwaj, A. et al. (2013). Digital Business Strategy: Toward a Next Generation of Insights. MIS Quarterly, 37(2), 471-482.

Bi, R. et al. (2011). Building IT Capability to Increase Organizational Performance: A Path-Oriented Process. Pacific Asia Journal of the Association for Information Systems, 3(3).

Blondiau, A. et al. (2013). Design and Implementation Challenges of Maturity Models for Healthcare Organizations $\mathbb{X}$ An Experience Report. Proceedings of the 16th International Symposium on Health Information Management Research, 1-9.

Bourreau, M. et al. (2012). The Impact of a Radical Innovation on Business Models: Incremental Adjustments or Big Bang? Industry and Innovation, 19(5), 415-435.

Brenner, W. et al. (2014). User, Use \& Utility Research. Business \& Information Systems Engineering, 56(1), 65-72.

Brynjolfsson, E./Hitt, L. M. (2000). Beyond Computation: Information Technology, Organizational Transformation and Business Performance. The Journal of Economic Perspectives, 14(4), 23-48.

Buchwald, A. et al. (2014). Business value through controlled IT: toward an integrated model of IT governance success and its impact. Journal of Information Technology, 29(2), 128-147.

Chae, H.-C., et al. (2014). Information Technology Capability and Firm Performance: Enabling and Facilitating Roles. MIS Quarterly, 38(1), 305-326.

Chaffey, D. (2010). Applying organisational capability models to assess the maturity of digital-marketing governance. Journal of Marketing Management, 26(3/4), 187-196.

Chakravarty, A. et al. (2013). Information Technology Competencies, Organizational Agility, and Firm Performance: Enabling and Facilitating Roles. Information Systems Research, 24(4), 976 997.

Chatterjee, D./Ravichandran, T. (2013). Governance of Interorganizational Information Systems: A Resource Dependence Perspective. Information Systems Research, 24(2), 261-278.

Chen, D. Q. et al. (2010). Information Systems Strategy: Reconceptualization, Measurement, and Implications. MIS Quarterly, 34(2), 233-259.

Cleven, A. et al. (2014). Process management in hospitals: an empirically grounded maturity model. Business Research, 1-26.

Cravens, D. W. et al. (2009). Management framework guiding strategic thinking in rapidly changing markets. Journal of Marketing Management, 25(1/2), 31-49. 
De Bruin, T. et al. (2005). Understanding the Main Phases of Developing a Maturity Assessment Model. In Australasian Conference on Information Systems (ACIS),. Australasian Chapter of the Association for Information Systems.

DeVellis, R. F. (2003). Scale Development: theory and applications (2nd ed.). Thousand Oaks, CA Sage 2003.

Drnevich, P. L./Croson, D. C. (2013). Information Technology and Business-Level Strategy: Toward an Integrated Theoretical Perspective. MIS Quarterly, 37(2), 483-509.

Eisenhardt, K. M./Martin, J. A. (2000). Dynamic Capabilites: What are They? Strategic Management Journal, 21(10), 1105-1121.

Enkel, E. et al. (2011). Open Innovation Maturity Framework. International Journal of Innovation Management, 15(06), 1161-1189.

Frankenberger, K. et al. (2013). Network configuration, customer centricity, and performance of open business models: A solution provider perspective. Industrial Marketing Management, 42(5), 671-682.

Friedel, D./Back, A. (2012). Bestimmung des Enterprise-2.0-Entwicklungsstands mit einem Reifegradmodell. In Back, A. et al. (Eds.), Web 2.0 und Social Media in der Unternehmenspraxis Grundlagen, Anwendungen und Methoden mit zahlreichen Fallstudien (pp. 102-108). München: Oldenbourg.

Gal, U. et al. (2007). Explaining Radical Technology-Based Innovations in Organizations. In AMCIS 2007 Proceedings (Paper 113).

Ganguly, A. et al. (2009). Evaluating agility in corporate enterprises. International Journal of Production Economics, 118(2), 410-423.

Govindarajan, V./Kopalle, P. K. (2006). Disruptiveness of innovations: measurement and an assessment of reliability and validity. Strategic Management Journal, 27(2), 189-199.

Gray, P. et al. (2013). Realizing Strategic Value Through Center-Edge Digital Transformation in Consumer-Centric Industries. MIS Quarterly Executive, 12(1), 1-17.

Green, W./Cluley, R. (2014). The field of radical innovation: Making sense of organizational cultures and radical innovation. Industrial Marketing Management, 43(8), 1343-1350.

Grover, V./Kohli, R. (2012). Cocreating IT Value: New Capabilities and Metrics for Multifirm Environments. MIS Quarterly, 36(1), 225-232.

Hansen, A. M. et al. (2011). Rapid Adaptation in Digital Transformation: A Participatory Process for Engaging IS and Business Leaders. MIS Quarterly Executive, 10(4), 175-185.

Harris, J. et al. (2012). IT consumerization: when gadgets turn into enterprise IT tools. MIS Quarterly Executive, 2012(3), 99-112.

Henderson, J. C./Venkatraman, H. (1993). Strategic alignment: Leveraging information technology for transforming organizations. IBM Systems Journal, 32(1), 472-484.

Henfridsson, O./Yoo, Y. (2014). The liminality of trajectory shifts in institutional entrepreneurship. Organization Science, 25(3), 932-950.

Iivari, J./Iivari, N. (2011). Varieties of user-centredness: an analysis of four systems development methods. Information Systems Journal, 21(2), 125-153.

Jahani, B. et al. (2010). Measurement of enterprise architecture readiness within organizations. Business Strategy Series, 11(3), 177-191.

Janssen, S. et al. (2011). Using performance measures conceptually in innovation control. Journal of Management Control, 22(1), 107-128. 
Labusch, N. et al. (2014). A Reference Model for the Information-Based Support of Enterprise Transformations. In Tremblay, M. C. et al. (Eds.), Advancing the Impact of Design Science: Moving from Theory to Practice - 9th International Conference, DESRIST 2014, Miami, FL, USA, May 22-24, 2014. Proceedings (Vol. 8463, pp. 194-208). Springer International Publishing.

Lahrmann, G. et al. (2011). Inductive Design of Maturity Models: Applying the Rasch Algorithm for Design Science Research. In DESRIST 2011 (pp. 176-191).

Land, K.-H. (2014). Digital Readiness Index 2014: Executive Summary. Köln. Retrieved from http:// www.neuland.me/wp-content/uploads/2014/02/nl_Summary_v 5.pdf.

Leidner, D. E. et al. (2011). An empirical investigation of the relationship of IS strategy with firm performance. The Journal of Strategic Information Systems, 20(4), 419-437.

Leimeister, J. M. et al. (2009). Leveraging Crowdsourcing: Activation-Supporting Components for IT-Based Ideas Competition. Journal of Management Information Systems, 26(1), 197-224.

Leimeister, J. M. et al. (2014). Digital services for consumers. Electronic Markets, 24(4), 255-258.

Liang, T./Tanniru, M. (2006). Customer-Centric Information Systems. Journal of Management Information Systems, 23(3), 9-15.

Liu, D.-Y. et al. (2011). Resource fit in digital transformationLessons learned from the CBC Bank global e-banking project. Management Decision, 49(10), 1728-1742.

Lloyds Bank (2014). UK Business Digital Index 2014. Retrieved from: https://resources.lloydsbank.c om/news/launch-of-uk-business-digital-index-2014/.

Lyytinen, K./Rose, G. M. (2003). The Disruptive Nature of Information Technology Innovations: The Case of Internet Computing in Systems Development Organizations. MIS Quarterly, 27(4), 557-595.

Lyytinen, K. et al. (2010). Learning routines and disruptive technological change: Hyper-learning in seven software development organizations during internet adoption. Information Technology \& People, 23(2), 165-192.

Margherita, A./Petti, C. (2010). ICT-enabled and process-based change: an integrative roadmap. Business Process Management Journal, 16(3), 473-491.

Markides, C. (2006). Disruptive Innovation: In Need of Better Theory. Journal of Product Innovation Management, 23(1), 19-25.

Matt, C. et al. (2015). Digital Transformation Strategies. Business \& Information Systems Engineering, 57(5), 339-343.

Mettler, T. (2010). Supply Management im Krankenhaus: Konstruktion und Evaluation eines konfigurierbaren Reifegradmodells zur zielgerichteten Gestaltung. University of St.Gallen.

Mithas, S. et al. (2013). Leveraging Big Data and Business Analytics. IEEE IT Professional, 15(6), 18-20.

Mithas, S. et al. (2013). How a Firm's Competitive Environment and Digital Strategic Posture Influence Digital Business Strategy. MIS Quarterly, 37(2), 511-536.

Moreau, F. (2013). The Disruptive Nature of Digitization: The Case of the Recorded Music Industry. International Journal of Arts Management, 15(2), 18-31.

Mullaly, M. (2014). If maturity is the answer, then exactly what was the question? International Journal of Managing Projects in Business, 7(2), 169-185.

Neff, A. A. et al. (2014). Developing a maturity model for service systems in heavy equipment manufacturing enterprises. Information \& Management, 51(7), 895-911. 
Österle, H. (1995). Business Engineering: Prozess- und Systementwicklung. Springer-Verlag Berlin Heidelberg $\mathrm{GmbH}$.

Österle, H. (2007). Business Engineering - Geschäftsmodelle transformieren. In Loos, P./ Krcmar, H. (Eds.), Architekturen und Prozesse: Strukturen und Dynamik in Forschung und Unternehmen (pp. 71-84). Berlin: Springer.

Österle, H./Winter, R. (2003). Business Engineering. In Österle, H./ Winter, R. (Eds.), Business Engineering. Auf dem Weg zum Unternehmen des Informationszeitalters (pp. 3-19). Springer Berlin Heidelberg.

Otto, B. (2010). IT Governance and Organizational Transformation: Findings From an Action Research Study. In AMCIS 2010 Proceedings.

Paulk, M. C. et al. (1993). Capability maturity model for software, Version 1.1. Pittsburgh, Pennsylvania. Retrieved from http://onlinelibrary.wiley.com/doi/10.1002/0471028959.sof589/full.

Philip, G./McKeown, I. (2004). Business Transformation and Organizational Culture: The Role of Competency, IS and TQM. European Management Journal, 22(6), 624-636.

Pöppelbuß, J./Röglinger, M. (2011). What Makes a Useful Maturity Model? A Framework of General Design Principles for Maturity Models and Its Demonstration in Business Process Management. In ECIS 2011 Proceedings.

Pries-Heje, J./Baskerville, R. (2010). Management Design Theories. In Pries-Heje, J. et al. (Eds.), Human Benefit through the Diffusion of Information Systems Design Science Research SE - 16 (Vol. 318, pp. 263-281). Springer Berlin Heidelberg.

PwC. (2013). Profitable growth in the digital age: Unleash your potential. London. Retrieved from http://www.pwc.ch/user_content/editor/files/publ_adv/pwc_profitable_growth_e.pdf.

Qu, W. G. et al. (2010). The strategic value of IT insourcing: An IT-enabled business process perspective. The Journal of Strategic Information Systems, 19(2), 96-108.

Quaadgras, A. et al. (2014). Management commitments that maximize business impact from IT. Journal of Information Technology, 29(2), 114-127.

Raber, D. (2013). Reifegradmodellbasierte Weiterentwicklung von Business Intelligence im Unternehmen. University of St.Gallen.

Röglinger, M. et al. (2012). Maturity models in business process management. Business Process Management Journal, 18(2), 328-346.

Sambamurthy, V. et al. (2003). Shaping Agility through Digital Options: Reconceptualizing the Role of Information Technology in Contemporary Firms. MIS Quarterly, 27(2), 237-263.

Sammer, T. et al. (2013). The New Enterprise Mobility: Seizing the Opportunities and Challenges in Corporate Mobile IT. In Proceedings of the 19th Americas Conference on Information Systems (AMCIS) (pp. 1-8).

Sharma, R. et al. (2014). Transforming decision-making processes: a research agenda for understanding the impact of business analytics on organisations. European Journal of Information Systems, 23(4), 433-441.

Smith, H. A./McKeen, J. (2011). Enabling Collaboration with IT. Communications of the Association for Information Systems, 28(1).

Sonntag, R./Müller, M. (2013). E-Business Reifegradmodell. Der Wert von E-Business in Ihrem Unternehmen. Retrieved from: http://www.t-systems-mms.com/unternehmen/downloads/e-businessberatung.

Spee, A. P./Jarzabkowski, P. (2009). Strategy tools as boundary objects. Strategic Organization, London, UK, 7(2), 223-232. 
Steimel, B. et al. (2014). Digital Transformation Report 2014. Retrieved from http://www.dtaward.de/.

Swanson, E. B. (1994). Information Systems Among Innovation Organizations. Management Science, 40(9), 1069-1092.

Teece, D. J. et al. (1997). Dynamic Capabilities and Strategic Management. Strategic Management Journal, 18(7), 509-533.

Tiwana, A. et al. (2013). Special Issue: Information Technology and Organizational Governance: The IT Governance Cube. Journal of Management Information Systems, 30(3), 7-12.

Tornatzky, L. G. et al. (1990). The processes of technological innovation. Lexington (Mass.).

van Dyck, C. et al. (2005). Organizational Error Management Culture and Its Impact on Performance: A Two-Study Replication. Journal of Applied Psychology, 90(6), 1228-1240.

Walton, D. (2014). Social Systems Design in Organizational Change. In G. S. Metcalf (Ed.), Social Systems and Design SE - 9 (Vol. 1, pp. 213-232). Springer Japan.

Weichbrodt, J. et al. (2014). FlexWork-Phasenmodell: Ein Instrument zur Bestimmung der örtlichen und zeitlichen Arbeitsflexibilität in Organisationen. Retrieved from http://www.fhnw.ch/aps/ifk/ projekte/aktuelle-projekte/flex_work_phasenmodell/.

Weill, P./Woerner, S. L. (2013). The Future of the CIO in a Digital Economy. MIS Quarterly Executive, $12(2), 65-75$.

Westerman, G. et al. (2011). Digital Transformation: A Road-Map for Billion-Dollar Organizations. Cambridge MA \& Paris: MIT Center for Digital Business \& Capgemini Consulting.

Westerman, G. et al. (2012). The Digital Advantage: How digital leaders outperform their peers in every industry. Cambridge MA \& Paris: MIT Center for Digital Business \& Capgemini Consulting.

Wiklund, K. et al. (2013). Impediments in Agile Software Development: An Empirical Investigation. In Heidrich, J. et al. (Eds.), Product-Focused Software Process Improvement (pp. 35-49). Springer Berlin Heidelberg.

Winter, R. (2011). Business Engineering Navigator. Gestaltung und Analyse von Geschäftslösungen "Business-to-IT". Springer.

Yoo, Y. et al. (2010). The New Organizing Logic of Digital Innovation: An Agenda for Information Systems Research. Information Systems Research.

Zephir, O. et al. (2011). A maturity model to assess organisational readiness for change. International Journal of Technology Management, 55(3), 286-296.

\section{Anhang A}

Kriterienkatalog mit allen Dimensionen, Reifekriterien und zugehörigen Indikatoren

\begin{tabular}{|l|l|l|}
\hline & $\begin{array}{l}\text { Dimension / } \\
\text { Reifekriterium }\end{array}$ & Indikatoren \\
\hline $\mathbf{1}$ & Customer Experience & \multicolumn{2}{|l|}{} \\
\hline CX_1 & Analytics & $\begin{array}{l}\text { Um die Wirkung der Kanäle auf Kommunikations- und } \\
\text { Vertriebsziele zu analysieren, führen wir Kunden- und } \\
\text { Interaktionsdaten kanalübergreifend zusammen. }\end{array}$ \\
\hline CX_2 & Analytics & $\begin{array}{l}\text { Die Auswertung grosser Mengen an Kundendaten ge- } \\
\text { schieht automatisiert und in Echtzeit. }\end{array}$ \\
\hline
\end{tabular}




\begin{tabular}{|c|c|c|}
\hline & $\begin{array}{l}\text { Dimension / } \\
\text { Reifekriterium }\end{array}$ & Indikatoren \\
\hline CX_3 & Experience Design & $\begin{array}{l}\text { Angebote und Informationen sind in digitalen Kanälen } \\
\text { auf den Kundenkontext abgestimmt (z.B. je nach Situa- } \\
\text { tion im Verkaufsprozess). }\end{array}$ \\
\hline CX_4 & Experience Design & $\begin{array}{l}\text { Zentrale Kundenprozesse sind über alle relevanten digi- } \\
\text { talen und physischen Kanäle abgebildet und der Kunde } \\
\text { hat die Kanalwahl. }\end{array}$ \\
\hline CX_5 & Experience Design & $\begin{array}{l}\text { Interaktion und Design sind für den jeweiligen digitalen } \\
\text { Kanal optimiert. }\end{array}$ \\
\hline 2 & \multicolumn{2}{|l|}{ Produktinnovation } \\
\hline PI_1 & Geschäftsfelderweiterung & $\begin{array}{l}\text { Für Produkte und Dienstleistungen wurden durch digi- } \\
\text { tale Technologien neue Begleitangebote und damit ein } \\
\text { Mehrwert für den Kunden geschaffen. }\end{array}$ \\
\hline PI_2 & Geschäftsfelderweiterung & $\begin{array}{l}\text { In den vergangenen Jahren wurden neue digitale Ge- } \\
\text { schäftsmodelle und -ideen erfolgreich umgesetzt. }\end{array}$ \\
\hline PI_3 & Innovationsgrad & $\begin{array}{l}\text { Für die Entwicklung von innovativen digitalen Pro- } \\
\text { dukt-, Service- und Prozessideen wurden strategische } \\
\text { Rahmenbedingungen geschaffen. }\end{array}$ \\
\hline PI_4 & Innovationsgrad & $\begin{array}{l}\text { Unsere Mitarbeiter bringen aktiv neue digital getriebene } \\
\text { Produktideen ein, die in mehreren Fällen bereits umge- } \\
\text { setzt wurden. }\end{array}$ \\
\hline PI_5 & Digitale Kundenintegration & $\begin{array}{l}\text { Die Entwicklung von neuen digital getriebenen Pro- } \\
\text { duktideen durch Kunden wird aktiv gefördert. }\end{array}$ \\
\hline PI_6 & Digitale Kundenintegration & $\begin{array}{l}\text { Kunden werden systematisch zu ihrer Zufriedenheit } \\
\text { und Verbesserungsvorschlägen für digitale Kanäle be- } \\
\text { fragt. }\end{array}$ \\
\hline 3 & \multicolumn{2}{|l|}{ Strategie } \\
\hline ST_1 & Strategische Innovation & $\begin{array}{l}\text { Wir werden von Mitbewerbern und Fachkreisen als } \\
\text { Treiber von digitalen Innovationen wahrgenommen. }\end{array}$ \\
\hline ST_2 & Strategische Innovation & $\begin{array}{l}\text { Digitale Innovation wird systematisch vorangetrieben, } \\
\text { z.B. im Rahmen des jährlichen Strategiereviews. }\end{array}$ \\
\hline ST_3 & Strategische Innovation & $\begin{array}{l}\text { Strategische Optimierungen erfolgen zum grossen Teil } \\
\text { datenbasiert. }\end{array}$ \\
\hline ST_4 & Digitales Commitment & $\begin{array}{l}\text { "Digital Business" hat in unserer Gesamtstrategie einen } \\
\text { hohen Stellenwert und ist breit kommuniziert. }\end{array}$ \\
\hline ST_5 & Digitales Commitment & $\begin{array}{l}\text { Wir setzen uns aktiv damit auseinander, welche strategi- } \\
\text { schen Ressourcen der Firma auch im digitalen Zeitalter } \\
\text { ihren Wert behalten. }\end{array}$ \\
\hline ST_6 & Digitales Commitment & $\begin{array}{l}\text { Digitale Projekte stellen einen grossen Anteil im Pro- } \\
\text { jektportfolio dar und werden mit hoher Priorität voran- } \\
\text { getrieben. }\end{array}$ \\
\hline
\end{tabular}




\begin{tabular}{|c|c|c|}
\hline & $\begin{array}{l}\text { Dimension / } \\
\text { Reifekriterium }\end{array}$ & Indikatoren \\
\hline $\mathrm{ST}_{-} 7$ & Digitales Commitment & $\begin{array}{l}\text { Die digitale Geschäftstransformation ist ein laufendes } \\
\text { strategisches Change-Projekt in unserer gesamten Un- } \\
\text { ternehmung. }\end{array}$ \\
\hline 4 & \multicolumn{2}{|l|}{ Organisation } \\
\hline OR_1 & Digitale Teamaufstellung & $\begin{array}{l}\text { Digitale Projekte werden von Anfang an abteilungs- } \\
\text { und funktionsübergreifend organisiert und umgesetzt. }\end{array}$ \\
\hline OR_2 & Digitale Teamaufstellung & $\begin{array}{l}\text { Es gibt eine kanalübergreifende Führung und Verant- } \\
\text { wortung für Markenführung, Umsatz und Kundenzu- } \\
\text { friedenheit. }\end{array}$ \\
\hline OR_3 & Unternehmerische Agilität & $\begin{array}{l}\text { Unsere Geschäftsstrategie zielt darauf ab, neue Fähig- } \\
\text { keiten aufzubauen, um in vielen denkbaren digitalen } \\
\text { Szenarien erfolgreich zu sein. }\end{array}$ \\
\hline OR_4 & Unternehmerische Agilität & $\begin{array}{l}\text { Es existiert eine systematische "Frühwarnung" zur } \\
\text { Identifikation neuer Technologien und Geschäftsmodel- } \\
\text { le mit Relevanz für unser Geschäft. }\end{array}$ \\
\hline OR_5 & Partnernetzwerk & $\begin{array}{l}\text { Es existiert ein Partnernetzwerk mit externen Spezial- } \\
\text { dienstleistern. }\end{array}$ \\
\hline OR_6 & Partnernetzwerk & $\begin{array}{l}\text { Mit externen Partnern gibt es standardisierte Prozesse } \\
\text { in der Zusammenarbeit. }\end{array}$ \\
\hline 5 & \multicolumn{2}{|l|}{ Prozessdigitalisierung } \\
\hline PR_1 & Touchpoint Management & $\begin{array}{l}\text { Digitale und mobile Kanäle sowie Social-Media-Platt- } \\
\text { formen sind konsequent in Vertriebs- und Transaktions- } \\
\text { prozesse integriert. }\end{array}$ \\
\hline PR_2 & Touchpoint Management & $\begin{array}{l}\text { Für digitale und mobile Touchpoints sind Qualitäts- } \\
\text { merkmale und Ziele definiert, die regelmässig überprüft } \\
\text { werden. }\end{array}$ \\
\hline PR_3 & Automatisierung & $\begin{array}{l}\text { Kernprozesse der Leistungserstellung werden regelmäs- } \\
\text { sig auf Optimierungspotenziale durch digitale Techno- } \\
\text { logien überprüft und unter Berücksichtigung der Wirt- } \\
\text { schaftlichkeit automatisiert. }\end{array}$ \\
\hline PR_4 & Automatisierung & $\begin{array}{l}\text { Das digitale Potenzial in Kernprozessen ist in Bezug auf } \\
\text { aktuelle Best Practices ausgeschöpft, umgesetzt und eta- } \\
\text { bliert. }\end{array}$ \\
\hline PR_5 & $\begin{array}{l}\text { Digitale Marketingkommu- } \\
\text { nikation }\end{array}$ & $\begin{array}{l}\text { Digitale und mobile Kanäle sowie Social-Media-Platt- } \\
\text { formen sind konsequent in Kommunikations- und Ser- } \\
\text { viceprozesse integriert. }\end{array}$ \\
\hline PR_6 & $\begin{array}{l}\text { Digitale Marketingkommu- } \\
\text { nikation }\end{array}$ & $\begin{array}{l}\text { Digitale Kommunikationsmassnahmen werden auf Ba- } \\
\text { sis von Analytics umgesetzt. }\end{array}$ \\
\hline PR_7 & $\begin{array}{l}\text { Digitale Marketingkommu- } \\
\text { nikation }\end{array}$ & $\begin{array}{l}\text { Die Ausgaben für digitale Kommunikation richten sich } \\
\text { nach der Mediennutzung der Kunden und dem Leis- } \\
\text { tungsbeitrag des jeweiligen Kanals. }\end{array}$ \\
\hline 6 & \multicolumn{2}{|l|}{ Zusammenarbeit } \\
\hline
\end{tabular}




\begin{tabular}{|c|c|c|}
\hline & $\begin{array}{l}\text { Dimension / } \\
\text { Reifekriterium }\end{array}$ & Indikatoren \\
\hline ZU_1 & Kollaboration & $\begin{array}{l}\text { Digitale Lösungen zum Informationsaustausch und } \mathrm{Zu} \text { - } \\
\text { sammenarbeit sind bei uns intern und extern in regem } \\
\text { Einsatz (z.B. Jive, Confluence etc.). }\end{array}$ \\
\hline ZU_2 & Kollaboration & $\begin{array}{l}\text { Unified Communication mit Video-Conferencing und } \\
\text { Screensharing ist Standard in der internen und externen } \\
\text { Kommunikation (zB. Lync, Skype etc.). }\end{array}$ \\
\hline ZU_3 & Wissensmanagement & $\begin{array}{l}\text { Innovative Umsetzungen werden an Tagungen und } \\
\text { Konferenzen vorgestellt mit dem Ziel, Wissen in der } \\
\text { Community zu verbreiten. }\end{array}$ \\
\hline ZU_4 & Wissensmanagement & $\begin{array}{l}\text { Für digitale Themen sind interne Experten definiert und } \\
\text { wirken sowohl intern als auch extern als Ansprechpart- } \\
\text { ner (z.B. Practice Leads). }\end{array}$ \\
\hline ZU_5 & Flexibles Arbeiten & $\begin{array}{l}\text { Mitarbeitende, für die es sinnvoll ist, haben die Mög- } \\
\text { lichkeit, zuhause und mobil mit vollem Datenzugriff zu } \\
\text { arbeiten. }\end{array}$ \\
\hline ZU_6 & Flexibles Arbeiten & $\begin{array}{l}\text { Unser Unternehmen hat viel Erfahrung mit mobilem } \\
\text { Arbeiten, so dass kaum formale Regelungen nötig sind. }\end{array}$ \\
\hline 7 & \multicolumn{2}{|c|}{ ICT-Betrieb und Entwicklung } \\
\hline IT_1 & Agile Projektabwicklung & $\begin{array}{l}\text { Agile Methoden sind in der Projektabwicklung Stan- } \\
\text { dard (z.B. Scrum). }\end{array}$ \\
\hline IT_2 & Agile Projektabwicklung & $\begin{array}{l}\text { Wir sind in der Lage, für digitale Lösungen kurzfristig } \\
\text { Updates zu machen (mindestens innerhalb von } 6 \text { Wo- } \\
\text { chen). }\end{array}$ \\
\hline IT_3 & Agile Projektabwicklung & $\begin{array}{l}\text { Wir können neue digitale Produkte und Services an- } \\
\text { hand von Prototypen schnell testen und modifizieren. }\end{array}$ \\
\hline IT_4 & Integrierte Architektur & $\begin{array}{l}\text { Wir können neue digitale Angebote über definierte APIs } \\
\text { schnell an bestehende interne Systeme anbinden. }\end{array}$ \\
\hline IT_5 & Integrierte Architektur & $\begin{array}{l}\text { Daten zu Angeboten, Kunden und Transaktionen wer- } \\
\text { den zentral und kanalübergreifend geführt. }\end{array}$ \\
\hline IT_6 & Integrierte Architektur & $\begin{array}{l}\text { Unsere IT-Infrastruktur wird regelmässig auf die digita- } \\
\text { len Anforderungen hin überprüft und falls nötig zeitnah } \\
\text { erneuert. }\end{array}$ \\
\hline IT_7 & IT-Expertise & $\begin{array}{l}\text { Digitale Schlüsseltechnologien für die jeweilige Branche } \\
\text { sind definiert und können von unserer internen IT ge- } \\
\text { führt werden. }\end{array}$ \\
\hline IT_8 & IT-Expertise & $\begin{array}{l}\text { Wir haben eine klare Strategie zur Beschaffung oder Im- } \\
\text { plementierung digitaler Schlüsseltechnologien. }\end{array}$ \\
\hline 8 & \multicolumn{2}{|l|}{ Kultur und Expertise } \\
\hline KU_1 & Digitale Affinität & $\begin{array}{l}\text { Unsere Mitarbeitenden wenden für ihre Aufgaben nütz- } \\
\text { liche digitale Lösungen an. }\end{array}$ \\
\hline
\end{tabular}




\begin{tabular}{|c|c|c|}
\hline & $\begin{array}{l}\text { Dimension / } \\
\text { Reifekriterium }\end{array}$ & Indikatoren \\
\hline KU_2 & Digitale Affinität & $\begin{array}{l}\text { Bei der Rekrutierung von neuen Mitarbeitenden sind } \\
\text { die digitalen Fähigkeiten ein wichtiges Auswahlkriteri- } \\
\text { um. }\end{array}$ \\
\hline KU_3 & Digitale Affinität & $\begin{array}{l}\text { Unsere Mitarbeitenden sind mit unseren digitalen Ange- } \\
\text { boten vertraut und wenden diese selbst an. }\end{array}$ \\
\hline KU_4 & Risikobereitschaft & $\begin{array}{l}\text { Unser Unternehmen ist bereit, eigene Angebote oder } \\
\text { Prozesse zu substituieren, wenn es die digitale Transfor- } \\
\text { mation erfordert. }\end{array}$ \\
\hline KU_5 & Risikobereitschaft & $\begin{array}{l}\text { Das Ausprobieren von neuen Ideen wird trotz mögli- } \\
\text { chen Scheiterns gefördert. }\end{array}$ \\
\hline KU_6 & Fehlerkultur & $\begin{array}{l}\text { Fehler und Lehren aus gescheiterten Projekten werden } \\
\text { nicht verschwiegen, sondern proaktiv kommuniziert. }\end{array}$ \\
\hline KU_7 & Fehlerkultur & $\begin{array}{l}\text { Wir werten gemachte Fehler aus, um unsere Prozesse } \\
\text { und Lösungen zu verbessern. }\end{array}$ \\
\hline 9 & \multicolumn{2}{|l|}{ Transformationsmanagement } \\
\hline TR_1 & Governance & $\begin{array}{l}\text { Wir haben eine digitale Roadmap erarbeitet, die zur di- } \\
\text { gitalen Transformation unseres Unternehmens einge- } \\
\text { setzt wird. }\end{array}$ \\
\hline TR_2 & Governance & $\begin{array}{l}\text { Wir haben Rollen, Verantwortlichkeiten und Entschei- } \\
\text { dungsprozesse für die digitale Transformation definiert. }\end{array}$ \\
\hline TR_3 & $\begin{array}{l}\text { Management-Unterstüt- } \\
\text { zung }\end{array}$ & $\begin{array}{l}\text { Geschäftsleitung und Verwaltungsrat erkennen die } \\
\text { Wichtigkeit von Digital Business und stellen entspre- } \\
\text { chende Ressourcen zur Verfügung. }\end{array}$ \\
\hline TR_4 & $\begin{array}{l}\text { Management-Unterstüt- } \\
\text { zung }\end{array}$ & $\begin{array}{l}\text { Das mittlere Management erkennt die Wichtigkeit von } \\
\text { Digital Business und treibt in hohem Masse die Umset- } \\
\text { zung von digitalen Projekten. }\end{array}$ \\
\hline TR_5 & $\begin{array}{l}\text { Management-Unterstüt- } \\
\text { zung }\end{array}$ & $\begin{array}{l}\text { Eine bereichsübergreifende Verantwortung für die digi- } \\
\text { tale Transformation ist auf der obersten Führungsebene } \\
\text { verankert. }\end{array}$ \\
\hline TR_6 & Performance Measurement & $\begin{array}{l}\text { Leistungsindikatoren in Bezug auf operative Ziele des } \\
\text { Digital Business sind in Management-Zielvereinbarun- } \\
\text { gen verankert. }\end{array}$ \\
\hline TR_7 & Performance Measurement & $\begin{array}{l}\text { Die Ziele der digitalen Transformation sind messbar de- } \\
\text { finiert und werden periodisch überprüft. }\end{array}$ \\
\hline
\end{tabular}

Tabelle 3: Kriterienkatalog 


\section{Anhang B}

Übersicht über die Teilnehmer der qualitativen Erhebung:

\begin{tabular}{|c|c|c|c|}
\hline Position & Branche & Erfahrung & Teilnahme \\
\hline $\begin{array}{l}\text { Leiter Digital Business } \\
\text { Applications }\end{array}$ & Versicherungen & k.A. & Telefoninterview \\
\hline Leiter Mobile IT & Transport / Logistik & k.A. & Telefoninterview \\
\hline $\begin{array}{l}\text { Leiter Digital Business Trans- } \\
\text { formation }\end{array}$ & Versicherungen & > 20 Jahre & Telefoninterview \\
\hline Director Online & Telekommunikation & $>10$ Jahre & Telefoninterview \\
\hline Managing Director AT \& CH & Banken & > 15 Jahre & Telefoninterview $^{2}$ \\
\hline $\begin{array}{l}\text { Business Development Mana- } \\
\text { ger }\end{array}$ & Banken & $>10$ Jahre & Telefoninterview2 \\
\hline Leiter E-Business & Transport / Logistik & > 15 Jahre & Telefoninterview \\
\hline Leiter Marketing & Telekommunikation & $>20$ Jahre & Fokusgruppe 1 \\
\hline Leiter Business Prozesse & Detailhandel & $>20$ Jahre & Fokusgruppen $1 / 2$ \\
\hline Managing Director & Verwaltung / Schulen & $>15$ Jahre & Fokusgruppen $1 / 2$ \\
\hline Digital Transformation Lead & Transport / Logistik & $>20$ Jahre & Fokusgruppe 1 \\
\hline Geschäftsführer & Medien / Verlage & $>20$ Jahre & Fokusgruppen $1 / 2$ \\
\hline Head of Online Management & Transport / Logistik & $>15$ Jahre & Fokusgruppen $1 / 2$ \\
\hline Head of IT Strategy & Versicherungen & $>10$ Jahre & Fokusgruppe 1 \\
\hline Managing Partner & Beratung & $>15$ Jahre & Fokusgruppen $1 / 2$ \\
\hline Innovation Strategist & Banken & $>10$ Jahre & Fokusgruppe 1 \\
\hline Strategy \& Innovation Lead & Optik / Elektronik & $>15$ Jahre & Fokusgruppen $1 / 2$ \\
\hline Leiter Fachbereich & Verwaltung / Schulen & $>20$ Jahre & Fokusgruppe 1 \\
\hline
\end{tabular}

Tabelle 4: Übersicht über Teilnehmer an Experteninterview und Fokusgruppe

2 Doppelinterview mit zwei Teilnehmern. 
Sabine Berghaus ist wissenschaftliche Mitarbeiterin und Doktorandin am Lehrstuhl von Prof. Dr. Andrea Back. Sie forscht am Competence Center Mobile Business am Institut für Wirtschaftsinformatik der HSG.

Anschrift: Institut für Wirtschaftsinformatik, Unterer Graben 21, CH-9000 St. Gallen, Tel.: +41 7122438 70, E-Mail: sabine.berghaus@unisg.ch

Andrea Back ist seit 1994 Professorin für Betriebswirtschaftslehre und Wirtschaftsinformatik an der Universität St. Gallen und Direktorin des Instituts für Wirtschaftsinformatik IWI-HSG. Sie leitet die Competence Center Mobile Business und Business 2.0.

Anschrift: Institut für Wirtschaftsinformatik, Unterer Graben 21, CH-9000 St. Gallen, Tel.: +41 7122425 45, E-Mail: andrea.back@unisg.ch 